\title{
Subspaces and Orthogonal Decompositions Generated by Bounded Orthogonal Systems
}

\author{
Olivier Guédon, Shahar Mendelson ${ }^{1}$, Alain Pajor \\ and Nicole Tomczak-Jaegermann ${ }^{2}$
}

\begin{abstract}
We investigate properties of subspaces of $L_{2}$ spanned by subsets of a finite orthonormal system bounded in the $L_{\infty}$ norm. We first prove that there exists an arbitrarily large subset of this orthonormal system on which the $L_{1}$ and the $L_{2}$ norms are close, up to a logarithmic factor. Considering for example the Walsh system, we deduce the existence of two orthogonal subspaces of $L_{2}^{n}$, complementary to each other and each of dimension roughly $n / 2$, spanned by \pm 1 vectors (i.e. Kashin's splitting) and in logarithmic distance to the Euclidean space. The same method applies for $p>2$, and, in connection with the $\Lambda_{p}$ problem (solved by Bourgain), we study large subsets of this orthonormal system on which the $L_{2}$ and the $L_{p}$ norms are close (again, up to a logarithmic factor).
\end{abstract}

AMS Subject Classification (2000). 46B07; 46B09; 42A05; 42A61; 94B75; 62 G99.

Keywords. Empirical processes, generic chaining, bounded orthogonal systems, orthogonal decompositions.

\section{Introduction}

In this note we consider a space $L_{2}$ of functions on a probability space and we investigate properties of its subspaces spanned by a finite subset of an orthonormal system which consists of functions bounded in $L_{\infty}$. Typical examples of such systems are the trigonometric and the Walsh systems.

The question we study is whether there is a subspace spanned by a large subset of the orthonormal system on which the $L_{2}$ and $L_{p}$ norms are close. The two cases we focus on are when $p>2$ and $p=1$. Formally we address

Question 1. Let $\left(\varphi_{j}\right)_{j=1}^{n}$ be an orthonormal system in $L_{2}$ with $\left\|\varphi_{j}\right\|_{L_{\infty}} \leq K$.

\footnotetext{
${ }^{1}$ Partially supported by an Australian Research Council Discovery grant.

${ }^{2}$ This author holds the Canada Research Chair in Geometric Analysis.
} 
1. Let $1 \leq k<n$. Is there a subset $I \subset\{1, \ldots, n\}$ of cardinality larger than $n-k$ and $A_{1}$, such that for every $\left(a_{i}\right)_{i=1}^{n} \in \mathbb{C}^{n}$,

$$
\left(\sum_{i \in I}\left|a_{i}\right|^{2}\right)^{1 / 2} \leq A_{1}\left\|\sum_{i \in I} a_{i} \varphi_{i}\right\|_{L_{1}} ?
$$

2. For $p>2$ is there a large set $J \subset\{1, \ldots, n\}$ and $A_{p}>0$ such that for every $\left(a_{i}\right)_{i=1}^{n} \in \mathbb{C}^{n}$,

$$
\left(\sum_{j \in J}\left|a_{j}\right|^{2}\right)^{1 / 2} \geq A_{p}\left\|\sum_{j \in J} a_{j} \varphi_{j}\right\|_{L_{p}} ?
$$

The first part of Question 1 is connected with the problem of selecting a proportion of characters. Improving a result of Bourgain, Talagrand [Ta2] showed that there exists a set $I$ of cardinality proportional to $n$ (that is, $|I| \sim \delta_{0} n$, where $\delta_{0}>0$ is a small constant that depends on $\left.K\right)$ such that on the span of $\left(\varphi_{i}\right)_{i \in I}$, the $L_{1}$ and $L_{2}$ norms are equivalent with $A_{1} \leq C K(\log n \log \log n)^{1 / 2}$. Here we show that a similar result holds for every $1 \leq k<n$. More precisely, introducing the spaces $L_{p}^{n}(1 \leq p \leq \infty)$ in $(2.2)$ below, we prove:

Theorem A. Let $\left(\varphi_{j}\right)_{j=1}^{n}$ be an orthonormal system in $L_{2}^{n}$ with $\left\|\varphi_{j}\right\|_{L_{\infty}^{n}} \leq K$ for $1 \leq j \leq n$. For any $1 \leq k<n$ there exists a subset $I \subset\{1, \ldots, n\}$ with $|I| \geq n-k$ such that for every $a=\left(a_{i}\right) \in \mathbb{C}^{n}$,

$$
\left(\sum_{i \in I}\left|a_{i}\right|^{2}\right)^{1 / 2} \leq C K \sqrt{n / k} \sqrt{\log n}(\log (1+k))^{3 / 2}\left\|\sum_{i \in I} a_{i} \varphi_{i}\right\|_{L_{1}^{n}},
$$

where $C>0$ is a universal constant.

Although Talagrand proved a better estimate, it is applicable only when $n-k$ is a small proportion of $n$. Our result allows us to obtain a Kashin splitting of $\mathbb{C}^{2 n}$ spanned by a subset of a bounded orthonormal system. Recall that a Kashin splitting of $\mathbb{C}^{2 n}$ consists of two orthogonal subspaces $E_{0}, E_{1}$ of $\mathbb{C}^{2 n}$, both of dimension $n$, such that on $E_{0}$ and $E_{1}$ the $L_{1}^{2 n}$ and $L_{2}^{2 n}$ norms are equivalent.

In other words, there is an absolute constant $c$ (independent of $n$ ) such that for every $x \in E_{i}, i=0,1$,

$$
c\|x\|_{L_{2}^{2 n}} \leq\|x\|_{L_{1}^{2 n}} \leq\|x\|_{L_{2}^{2 n}} .
$$

We show in Theorem 2.4 that if $\left(\varphi_{i}\right)_{i=1}^{n}$ is a bounded orthonormal system as above, then we can find a subset $I$ of $\{1, \cdots, n\}$, roughly of cardinality $n / 2$, such that, setting $I^{c}=\{1, \cdots, n\} \backslash I$, if $E_{0}=\operatorname{span}\left(\varphi_{i}\right)_{i \in I}$ and $E_{1}=\operatorname{span}\left(\varphi_{i}\right)_{i \in I^{c}}$, then the $L_{1}^{n}$ and $L_{2}^{n}$ are close on $E_{i}$. This applies in particular to the trigonometric or the Walsh system. In the latter case, it gives a Kashin splitting of $\mathbb{R}^{n}$ spanned by \pm 1 -valued vectors. 
The second part of Question 1 is connected with the $\Lambda_{p}$ problem which has been solved by Bourgain [B1]. He proved that if $\left(\varphi_{i}\right)_{i=1}^{n}$ is an orthonormal system of functions bounded by $K$ in $L_{\infty}$, then one can find $J \subset\{1, \ldots, n\},|J| \geq C(K) n^{2 / p}$, such that on the span of $\left(\varphi_{j}\right)_{j \in J}$ the $L_{2}$ and $L_{p}$ are equivalent (with an absolute constant). We will prove here the following

Theorem B. Let $\left(\varphi_{j}\right)_{j=1}^{n} \subset L_{2}$ be an orthonormal system bounded in $L_{\infty},\left\|\varphi_{j}\right\|_{L_{\infty}} \leq$ $K$ for $1 \leq j \leq n$. Let $2<p<\infty$. There exists a subset $J \subset\{1, \ldots, n\}$ of cardinality $|J| \geq c_{p} K^{4 / p} n^{2 / p}(\log n)^{3}$ such that for all scalars $a_{1}, \ldots a_{n}$,

$$
\left\|\sum_{j \in J} a_{j} \varphi_{j}\right\|_{L_{p}} \leq K C_{p}(\log n)^{3 / 2}\left(\sum_{j \in J} a_{j}^{2}\right)^{1 / 2}
$$

where $c_{p}$ and $C_{p}$ are constants depending only on $p$.

The estimates we present here do not reconstruct the full strength of Bourgain's solution. The equivalence constant that we obtain is slightly weaker, namely, $C(p, K) \log ^{3 / 2} n$, but the set $J$ is larger, with cardinality at least $C(p, K) n^{2 / p} \log ^{3} n$.

The proofs of all these estimates are simple and are based on arguments coming from the theory of empirical processes.

\section{Preliminaries}

We begin with a notational convention. Throughout, all constants are positive numbers denoted by $c, C$ etc. Their values can change from line to line. We require two preliminary results. The first one, Theorem 1.2 below, is an estimate on the supremum of the following empirical process defined on the probability space $(\Omega, \mu)$,

$$
\sup _{f \in F}\left|\frac{1}{k} \sum_{i=1}^{k} f^{2}\left(X_{i}\right)-\mathbb{E} f^{2}\right|,
$$

where $F$ is a class of functions and $X_{1}, \ldots, X_{k}$ are independent random variables distributed according to $\mu$. Using a symmetrization argument due to Giné and Zinn [GZ], it suffices to bound the Rademacher process $v \rightarrow \sum_{i=1}^{k} \varepsilon_{i} v_{i}$ indexed by the random projections of $F^{2}$, i.e., by the random sets

$$
P_{\sigma} F^{2}=\left\{\left(f^{2}\left(X_{1}\right), \ldots, f^{2}\left(X_{k}\right)\right): f \in F\right\} .
$$

Since a Rademacher process is subgaussian, one can analyze its behavior using the $\gamma_{2}$ functional of $P_{\sigma} F^{2}$ with respect to the random $\ell_{\infty}$ metric. This argument is due to Rudelson [R1] and the formulation below was implicit in [RV] or [GR]. We present it for the sake of completeness. 
Recall that for a metric space $(T, d)$, an admissible sequence of $T$ is a collection of subsets of $T,\left\{T_{s}: s \geq 0\right\}$, such that for every $s \geq 1,\left|T_{s}\right|=2^{2^{s}}$ and $\left|T_{0}\right|=1$.

Definition 1.1. [Ta1] For a metric space $(T, d)$, define

$$
\gamma_{2}(T, d)=\inf \sup _{t \in T} \sum_{s=0}^{\infty} 2^{s / 2} d\left(t, T_{s}\right)
$$

where the infimum is taken with respect to all admissible sequences of $T$.

Theorem 1.2. There exists an absolute constant $c$ for which the following holds. Let $X_{1}, \ldots, X_{k}$ be independent copies of $X$ and let

$$
d_{\infty, k}(f, g)=\max _{1 \leq i \leq k}\left|f\left(X_{i}\right)-g\left(X_{i}\right)\right|
$$

and set $U_{k}=\left(\mathbb{E} \gamma_{2}^{2}\left(F, d_{\infty, k}\right)\right)^{1 / 2}$ and $\sigma_{F}=\left(\sup _{f \in F} \mathbb{E} f^{2}(X)\right)^{1 / 2}$. Then,

$$
\mathbb{E} \sup _{f \in F}\left|\sum_{i=1}^{k}\left(f^{2}\left(X_{i}\right)-\mathbb{E} f^{2}(X)\right)\right| \leq c \max \left(\sqrt{k} \sigma_{F} U_{k}, U_{k}^{2}\right) .
$$

Proof. Set

$$
A:=\mathbb{E} \sup _{f \in F}\left|\sum_{i=1}^{k}\left(f^{2}\left(X_{i}\right)-\mathbb{E} f^{2}(X)\right)\right|,
$$

then by a symmetrization argument [GZ],

$$
A \leq \mathbb{E}_{X} \mathbb{E}_{\varepsilon} \sup _{f \in F}\left|\sum_{i=1}^{k} \varepsilon_{i} f^{2}\left(X_{i}\right)\right|,
$$

where $\left(\varepsilon_{i}\right)$ are iid $( \pm 1)$ Bernoulli random variables. Since the Rademacher process $\left(\sum_{i=1}^{k} \varepsilon_{i} f^{2}\left(X_{i}\right)\right)_{f \in F}$ is subgaussian with respect to the $\ell_{2}^{k}$ metric, it satisfies the subgaussian increment condition with respect to the random metric on $F$ defined by

$$
d(f, g)=\left(\sum_{i=1}^{k}\left(f^{2}\left(X_{i}\right)-g^{2}\left(X_{i}\right)\right)^{2}\right)^{1 / 2} .
$$

Therefore, by a generic chaining argument [Ta1] (see e.g., Theorem 1.2.6), for every $X_{1}, \ldots, X_{k}$,

$$
\mathbb{E}_{\varepsilon} \sup _{f \in F}\left|\sum_{i=1}^{k} \varepsilon_{i} f^{2}\left(X_{i}\right)\right| \leq c \gamma_{2}(F, d) .
$$

Recall that $d_{\infty, k}(f, g)=\max _{1 \leq i \leq k}\left|f\left(X_{i}\right)-g\left(X_{i}\right)\right|$, and thus for every $f, g \in F$,

$$
d(f, g) \leq 2 d_{\infty, k}(f, g) \sup _{f \in F}\left(\sum_{i=1}^{k} f^{2}\left(X_{i}\right)\right)^{1 / 2} .
$$


Combining this with the previous estimate we get, for every $X_{1}, \ldots, X_{k}$,

$$
\mathbb{E}_{\varepsilon} \sup _{f \in F}\left|\sum_{i=1}^{k} \varepsilon_{i} f^{2}\left(X_{i}\right)\right| \leq c \sup _{f \in F}\left(\sum_{i=1}^{k} f^{2}\left(X_{i}\right)\right)^{1 / 2} \gamma_{2}\left(F, d_{\infty, k}\right) .
$$

This implies that

$$
\mathbb{E}_{X} \mathbb{E}_{\varepsilon} \sup _{f \in F}\left|\sum_{i=1}^{k} \varepsilon_{i} f^{2}\left(X_{i}\right)\right| \leq c\left(\mathbb{E}_{X} \sup _{f \in F} \sum_{i=1}^{k} f^{2}\left(X_{i}\right)\right)^{1 / 2}\left(\mathbb{E}_{X} \gamma_{2}^{2}\left(F, d_{\infty, k}\right)\right)^{1 / 2}
$$

Since

$$
\mathbb{E}_{X} \sup _{f \in F} \sum_{i=1}^{k} f^{2}\left(X_{i}\right) \leq A+k \sup _{f \in F} \mathbb{E}_{X} f^{2}(X)
$$

we get

$$
A \leq c\left(A+k \sigma_{F}^{2}\right)^{1 / 2} U_{k}
$$

from which the claim easily follows.

The second preliminary result we require deals with the following situation. Let $n \geq 1$ and define a random variable $Y$ by $Y=j$ with probability $1 / n$, for $1 \leq j \leq n$. Let $Y_{1}, \ldots, Y_{n}$ be independent copies of $Y$ and for $1 \leq k \leq n$ set $\alpha_{k}:=\left|\left\{Y_{i}: 1 \leq i \leq k\right\}\right|$.

Lemma 1.3. For $1 \leq k \leq n, \mathbb{E} \alpha_{k}=n\left(1-(1-1 / n)^{k}\right)$. In particular, letting $\lambda:=k / n$,

$$
k \frac{1-e^{-\lambda}}{\lambda} \leq \mathbb{E} \alpha_{k} \leq k \frac{1-e^{-\lambda}+(e n)^{-\lambda}}{\lambda} .
$$

Furthermore, for $3 \leq k \leq n$ and every $0<\delta<1$,

$$
\mathbb{P}\left(\left|\alpha_{k}-\mathbb{E} \alpha_{k}\right| \geq \sqrt{\delta k / 2}\right) \leq 1 / \delta .
$$

Proof. The argument is straightforward. First, for $k \leq n$ it is easy to calculate the conditional expectation,

$$
\mathbb{E}\left(\alpha_{k} \mid \alpha_{k-1}\right)=\alpha_{k-1}^{2} / n+\left(\alpha_{k-1}+1\right)\left(1-\alpha_{k-1} / n\right)=1+\alpha_{k-1}(1-1 / n) .
$$

Thus $\mathbb{E} \alpha_{k}=1+(1-1 / n) \mathbb{E} \alpha_{k-1}$ implying the desired formula for $\mathbb{E} \alpha_{k}$, by iterating and noting that $\alpha_{1}=1$. The estimates for $\mathbb{E} \alpha_{k}$ easily follow.

Similarly, $\mathbb{E}\left(\alpha_{k}^{2} \mid \alpha_{k-1}\right)=1+(2-1 / n) \alpha_{k-1}+(1-2 / n) \alpha_{k-1}^{2}$, which yields

$$
\mathbb{E} \alpha_{k}^{2}=1+(2-1 / n) \mathbb{E} \alpha_{k-1}+(1-2 / n) \mathbb{E} \alpha_{k-1}^{2} .
$$

Thus, $\mathbb{E} \alpha_{k}^{2}-\left(\mathbb{E} \alpha_{k}\right)^{2}$ is equal to

$$
\begin{aligned}
& \left(1-\frac{2}{n}\right)\left(\mathbb{E} \alpha_{k-1}^{2}-\left(\mathbb{E} \alpha_{k-1}\right)^{2}\right)+\frac{1}{n} \mathbb{E} \alpha_{k-1}\left(1-\frac{1}{n} \mathbb{E} \alpha_{k-1}\right) \\
& \leq\left(1-\frac{2}{n}\right)\left(\mathbb{E} \alpha_{k-1}^{2}-\left(\mathbb{E} \alpha_{k-1}\right)^{2}\right)+\frac{1}{4} \leq\left(\mathbb{E} \alpha_{k-1}^{2}-\left(\mathbb{E} \alpha_{k-1}\right)^{2}\right)+\frac{1}{4}
\end{aligned}
$$


By iterating, it follows that for $k \geq 3,0 \leq \mathbb{E} \alpha_{k}^{2}-\left(\mathbb{E} \alpha_{k}\right)^{2} \leq 1+(k-1) / 4 \leq k / 2$. Chebyshev's inequality implies the required deviation estimate.

\section{Sections of $\ell_{1}^{n}$}

Here we use Theorem 1.2 to prove our results on uniformly bounded orthonormal systems in $L_{1}$.

In all our applications we will consider a probability space $(\Omega, \nu)$ and i.i.d. random variables $X_{1}, \ldots, X_{k}$ in $L_{\infty}$ (for $k=1,2, \ldots$ ) with $\left\|X_{i}\right\|_{L_{\infty}} \leq K$, where $K \geq 1$ is a fixed constant. In this setting define the semi-norm $\|\cdot\|_{X, k}$ on $L_{1}$ by

$$
\|y\|_{X, k}=\max _{1 \leq i \leq k}\left|\left\langle X_{i}, y\right\rangle\right| \quad \text { for } y \in L_{1} .
$$

The two main applications we present here are based on comparing the $L_{1}$ and $L_{2}$-norms on subspaces generated by subsets of bounded orthonormal systems, are done in a central (although slightly restricted) setting. Namely, we consider the space $\mathbb{C}^{n}$ as a function space on $\{1, \ldots, n\}$ equipped with the normalized counting measure and by $L_{p}^{n}$ (with $1 \leq p \leq \infty$ ) we denote the corresponding $L_{p}$ space, with the norm denoted by $\|\cdot\|_{L_{p}^{n}}$ and the inner product $\langle.,$.$\rangle . That is, for 1 \leq p<\infty$ and $y=\left(y_{i}\right) \in \mathbb{C}^{n}$,

$$
\|y\|_{L_{p}^{n}}=\left(\frac{1}{n} \sum_{i=1}^{n}\left|y_{i}\right|^{p}\right)^{1 / p} .
$$

By $B_{L_{p}^{n}}$ denote the unit ball in $L_{p}^{n}$. (The case $p=\infty$ is treated by a standard modification.)

Our first theorem in this section allows us to find subsets $I$ of $\{1, \ldots, n\}$ such that on the corresponding subspace of $L_{1}^{n}$, the norms from $L_{1}^{n}$ and from $L_{2}^{n}$ are comparable. Moreover, the cardinality of $I$ can be taken arbitrarily close to $n$.

Theorem 2.1. Let $\left(\varphi_{j}\right)_{j=1}^{n}$ be an orthonormal system in $L_{2}^{n}$ with $\left\|\varphi_{j}\right\|_{L_{\infty}^{n}} \leq K$ for $1 \leq j \leq n$. For any $1 \leq k<n$ there exists a subset $I \subset\{1, \ldots, n\}$ with $|I| \geq n-k$ such that for every $a=\left(a_{i}\right) \in \mathbb{C}^{n}$,

$$
\left(\sum_{i \in I}\left|a_{i}\right|^{2}\right)^{1 / 2} \leq C K \sqrt{n / k} \sqrt{\log n}(\log (1+k))^{3 / 2}\left\|\sum_{i \in I} a_{i} \varphi_{i}\right\|_{L_{1}^{n}}
$$

where $C>0$ is a universal constant.

Our theorem should be compared to a theorem by Talagrand and Bourgain ([Ta2], Theorem 1.5), which gives subsets $I$ of cardinality that is a sufficiently small proportion of $n$ (that is, $|I| \leq \delta_{0} n$, where $\delta_{0}>0$ depends on $K$ ), but with a better estimate $\sqrt{\log n \log \log n}$. Let us also note that for the trigonometric system, it is well known and easy to see from Szemerédi's theorem on arithmetic progressions $[\mathrm{Sz}]$, that for any subset $I$ whose cardinality is a fixed proportion of $n$ (say, $|I| \sim n / 4$ ), the upper bound must asymptotically go to infinity as $n \rightarrow \infty$. This follows by observing that it is so if a subset is an arithmetic progression. We 
have been informed by J. Bourgain that another argument shows that this upper bound involves a logarithmic term $\sqrt{\log n}$.

The proof of Theorem 2.1 requires the following upper bound of the $\gamma_{2}$ functional, obtained by entropy estimates. Recall that given bodies $K, B$ in $\mathbb{C}^{n}$ the covering number $N(K, B)$ is the smallest number of translates of $B$ needed to cover $K$. For a positive integer $\ell$ the entropy number $e_{\ell}(K, B)$ is the smallest $\varepsilon$ such that $N(K, \varepsilon B) \leq 2^{\ell-1}$. For a linear operator $T: X \rightarrow Y$ between two (finitedimensional) normed spaces $X, Y$ (with the unit balls $B_{X}, B_{Y}$, respectively), we let $e_{\ell}(T)=e_{\ell}\left(T\left(B_{X}\right), B_{Y}\right)$.

Lemma 2.2. There exist a constant $C>0$ such that for every $k \leq n$ and $X_{1}, \ldots, X_{k}$ with $\left\|X_{i}\right\|_{L_{\infty}^{n}} \leq K$,

$$
\gamma_{2}\left(B_{L_{1}^{n}},\|\cdot\|_{X, k}\right) \leq C K \sqrt{\log n}(\log (1+k))^{3 / 2} .
$$

Proof. Recall that by its definition, the $\gamma_{2}$ functional is bounded by an appropriate entropy integral (see [Ta1]). In turn, writing this integral in terms of entropy numbers of operators (see e.g. $[\mathrm{P}]$ chapter 5 ), and noting that the norms on $\ell_{\infty}^{n}$ and $L_{\infty}^{n}$ coincide, we get

$$
\gamma_{2}\left(B_{L_{1}^{n}},\|\cdot\|_{X, k}\right) \leq C \sum_{\ell=1}^{k} \frac{e_{\ell}\left(S^{*}\right)}{\sqrt{\ell}},
$$

where $C$ is an absolute constant, and the operator $S: \ell_{1}^{k} \rightarrow \ell_{\infty}^{n}$ is defined by $S\left(e_{i}\right)=X_{i}$ for every $i=1, \ldots, k$. Also, observe that although the above sum should be formally extended to infinity, the exponential decay of entropy numbers $e_{\ell}$ for $\ell$ larger than the rank of the operator, allows one to stop the summation at $\ell=k$.

The operator $S^{*}: \ell_{1}^{n} \rightarrow \ell_{\infty}^{k}$ satisfies $\left\|S^{*}\right\| \leq K$ and, by Proposition 3 of Carl $[\mathrm{C}]$, for $\ell \leq k$,

$$
e_{\ell}\left(S^{*}\right) \leq c K \sqrt{\frac{\log (1+k / \ell) \log (1+n / \ell)}{\ell}} .
$$

A simple computation completes the proof.

Proof of Theorem 2.1 Let $X$ be the random vector taking the value $\varphi_{i}$ with probability $1 / n$. Let $1 \leq k<n$ and let $X_{1}, \ldots, X_{k}$ be independent copies of $X$. We shall show that, with positive probability, the set of vectors $\left(\varphi_{i}\right)_{i \in I}$ defined by $\left\{\left(\varphi_{j}\right)_{j \leq n} \backslash\left(X_{i}\right)_{i=1}^{k}\right\}$ satisfies the required inequality.

Denote by $\Gamma$ the $k \times n$ matrix with rows $X_{1}, \ldots, X_{k}$, and thus, for $y \in \mathbb{C}^{n}$ $\Gamma y=\sum_{i=1}^{k}\left\langle X_{i}, y\right\rangle e_{i}$. For $y \in \mathbb{C}^{n}$ set $|y|=\left(\sum_{i=1}^{n}\left|y_{i}\right|^{2}\right)^{1 / 2}$ and put $S_{L_{2}^{n}}=\{y$ : $\left.\sum_{j=1}^{n}\left|y_{j}\right|^{2}=n\right\}$ to be the unit sphere in $L_{2}^{n}$. Note that for any star-shaped subset $T$ in $\mathbb{C}^{n}$ the following implication holds: whenever $\rho>0$ satisfies the inequality

$$
\sup _{y \in T \cap \rho S_{L_{2}^{n}}}\left|\sum_{i=1}^{k}\left(\left\langle X_{i}, y\right\rangle^{2}-\mathbb{E}\left\langle X_{i}, y\right\rangle^{2}\right)\right| \leq \frac{k \rho^{2}}{3 n}
$$


then

$$
\operatorname{diam}(\operatorname{ker} \Gamma \cap T) \leq \rho .
$$

Indeed, since $\mathbb{E}\left\langle X_{i}, y\right\rangle^{2}$ is constant $\left(=\rho^{2} / n\right)$ on $T \cap \rho S_{L_{2}^{n}}$, then condition (2.4) implies that for all $y \in T \cap \rho S_{L_{2}^{n}}$,

$$
\frac{2 k \rho^{2}}{3 n} \leq \sum_{i=1}^{k}\left\langle X_{i}, y\right\rangle^{2}=|\Gamma y|^{2} \leq \frac{4 k \rho^{2}}{3 n} .
$$

The homogeneity of (2.6) and the fact that $T$ is star-shaped imply that if the lower bound in (2.6) holds for all $y \in T \cap \rho S_{L_{2}^{n}}$, then the same lower bound also holds for all $y \in T$ with $\|y\|_{L_{2}^{n}} \geq \rho$. This in turn shows that if $y \in \operatorname{ker} \Gamma \cap T$ then $\|y\|_{L_{2}^{n}} \leq \rho$, as required in (2.5).

Finally note that since the $n \times n$ matrix whose rows are $\varphi_{1}, \ldots, \varphi_{n}$ is of course orthogonal, then the subspace $\operatorname{ker} \Gamma$ is spanned by the vectors $\left(\varphi_{i}\right)_{i \in I}=$ $\left\{\left(\varphi_{j}\right)_{j \leq n} \backslash\left(X_{i}\right)_{i=1}^{k}\right\}$. The cardinality of this set satisfies $|I| \geq n-k$, with the sharp inequality if some among values of $X_{1}, \ldots, X_{k}$ are equal. For $T=B_{L_{1}^{n}}$, this shows that whenever $\rho$ satisfies $(2.4)$, then

$$
\left\|\sum_{i \in I} a_{i} \varphi_{i}\right\|_{L_{2}^{n}} \leq \rho\left\|\sum_{i \in I} a_{i} \varphi_{i}\right\|_{L_{1}^{n}}
$$

for all scalars $a_{i}$, proving that (2.3) is satisfied with the constant $\rho$.

In order to find $\rho$ which satisfies (2.4) with positive probability, we use Theorem 1.2 and observe that $\sigma_{F}=\rho / \sqrt{n}$. It follows that

$$
\mathbb{E} \sup _{y \in B_{L_{1}^{n}} \cap \rho S_{L_{2}^{n}}}\left|\sum_{i=1}^{k}\left(\left\langle X_{i}, y\right\rangle^{2}-\mathbb{E}\left\langle X_{i}, y\right\rangle^{2}\right)\right| \leq c \max \left(\rho \sqrt{\frac{k}{n}} U_{k}, U_{k}^{2}\right)
$$

where $U_{k}=\left(\mathbb{E} \gamma_{2}^{2}\left(B_{L_{1}^{n}} \cap \rho S_{L_{2}^{n}},\|\cdot\|_{X, k}\right)\right)^{1 / 2}$. By Lemma 2.2,

$$
\gamma_{2}\left(B_{L_{1}^{n}} \cap \rho S_{L_{2}^{n}},\|\cdot\|_{X, k}\right) \leq \gamma_{2}\left(B_{L_{1}^{n}},\|\cdot\|_{X, k}\right) \leq c K \sqrt{\log n}(\log (1+k))^{3 / 2} .
$$

This gives an estimate for $U_{k}$ that we plug in the right-hand side of (2.7). To conclude, we use this estimate to show that for some numerical constant $C^{\prime}$ and for the choice of

$$
\rho=C^{\prime} K \sqrt{\log n}(\log (1+k))^{3 / 2} \sqrt{n / k},
$$

(2.4) holds with positive probability.

Remark 2.3. An analogous result to Theorem 2.1 also holds with probability close to 1 . For any $0<\delta^{\prime}<1$, first observe that by (2.7) and Chebyshev's inequality, for any $\rho^{\prime}>0$ we get a set of probability $\geq 1-\delta^{\prime}$ on which

$$
\sup _{y \in B_{L_{1}^{n}} \cap \rho^{\prime} S_{L_{2}^{n}}}\left|\sum_{i=1}^{k}\left(\left\langle X_{i}, y\right\rangle^{2}-\mathbb{E}\left\langle X_{i}, y\right\rangle^{2}\right)\right| \leq\left(c / \delta^{\prime}\right) \max \left(\rho^{\prime} \sqrt{\frac{k}{n}} U_{k}, U_{k}^{2}\right) .
$$


Setting $\rho^{\prime}:=c K \sqrt{\log n}(\log (1+k))^{3 / 2} / \sqrt{\delta} \delta^{\prime}$, a similar calculation as in the theorem above shows that on the set of probability $\geq 1-\delta^{\prime}$ (corresponding to $\rho^{\prime}$ ) we have an analogue of (2.4),

$$
\sup _{y \in B_{L_{1}^{n}} \cap \rho^{\prime} S_{L_{2}^{n}}}\left|\sum_{i=1}^{k}\left(\left\langle X_{i}, y\right\rangle^{2}-\mathbb{E}\left\langle X_{i}, y\right\rangle^{2}\right)\right| \leq \frac{k \rho^{\prime 2}}{3 n} .
$$

Again the same argument as before shows that on the same set we have

$$
\operatorname{diam}\left(\operatorname{ker} \Gamma \cap B_{L_{1}^{n}}\right) \leq \rho^{\prime}=c K \sqrt{\log n}(\log (1+k))^{3 / 2} / \sqrt{\delta} \delta^{\prime},
$$

as claimed.

Let us recall an important theorem concerning Euclidean sections of convex bodies, so-called Kashin's decomposition of $\ell_{1}^{2 k}([\mathrm{~K}]$, see also [S] for another proof and $[\mathrm{ST}],[\mathrm{P}]$ for important generalizations, primarily connected with the notion of volume ratio). Kashin's theorem says that $\mathbb{R}^{2 k}$ can be decomposed as a sum of two orthogonal subspaces $E_{0}, E_{1} \subset \mathbb{R}^{2 k}$ with $\operatorname{dim} E_{0}=\operatorname{dim} E_{1}=k$ on which the $L_{1}^{2 k}$ - and $L_{2}^{2 k}$-norms are equivalent, namely,

$$
(32 e \pi)^{-1}\|x\|_{L_{2}^{2 k}} \leq\|x\|_{L_{1}^{2 k}} \leq\|x\|_{L_{2}^{2 k}},
$$

for all $x \in E_{m}$ and $m=0,1$. The theorem below gives an analogous result on a decomposition of $L_{1}^{n}$ into two orthogonal subspaces with much more structure. To be more exact, the spaces are spanned by complementary subsets of a bounded orthonormal system in $L_{2}^{n}$, which are relatively close to a Euclidean space. Considering (a multiple of) the Walsh system (for $n=2^{\ell}$ ) we get two orthogonal subspaces of $L_{2}^{n}$ of dimension $\sim n / 2$ spanned by \pm 1 vectors and in the logarithmic distance to the Euclidean space. Let us recall again that for proportionaldimensional subspaces spanned by a subset of characters, the logarithmic term is necessary in general, at least for the trigonometric system.

Theorem 2.4. Let $\left(\varphi_{j}\right)_{j=1}^{n}$ be an orthonormal system in $L_{2}^{n}$ with $\left\|\varphi_{j}\right\|_{L_{\infty}^{n}} \leq K$ for $1 \leq j \leq n$. There exists a subset $I \subset\{1, \ldots, n\}$ with $n / 2-c^{\prime} \sqrt{n} \leq|I| \leq n / 2+c^{\prime} \sqrt{n}$ for some absolute constant $c^{\prime}>0$, such that for $m=0,1$ and every $a=\left(a_{i}\right) \in \mathbb{C}^{n}$,

$$
\left(\sum_{i \in J_{m}}\left|a_{i}\right|^{2}\right)^{1 / 2} \leq C K(\log n)^{2}\left\|\sum_{i \in J_{m}} a_{i} \varphi_{i}\right\|_{L_{1}^{n}}
$$

where $J_{0}=I$ and $J_{1}=I^{c}$ and $C>0$ is a universal constant.

Proof. Let $k=[\lambda n]$, with $\lambda:=\log 2$. We use the same notation and a part of the argument of the proof of Theorem 2.1. In particular, the random vectors $X_{1}, \ldots, X_{k}$ and the subset $I \subset\{1, \ldots, n\}$ are the same as before and $T=B_{L_{1}^{n}}$. It was shown that $(2.8)$ is satisfied with $\delta^{\prime}=1 / 4$ and probability $\geq 3 / 4$. Pick any realization of the $X_{i}$ 's that satisfies $(2.8)$ hence $(2.3)$ is valid i.e. $I$ satisfies inequality (2.9). Let $\Gamma$ be the same $k \times n$ matrix as before, we will show that

$$
\operatorname{diam}\left((\operatorname{ker} \Gamma)^{\perp} \cap T\right) \leq \rho^{\prime}
$$


by using a similar argument to $(2.5)$ with $\operatorname{ker} \Gamma$ replaced by $(\operatorname{ker} \Gamma)^{\perp}$. Hence, the set $I^{c}$ satisfies (2.9) as well. Thus having (2.8) satisfied with probability $\geq 3 / 4$, implies that with the same probability (2.9) will be satisfied for both $I$ and $I^{c}$. To complete the proof it will be then sufficient to note that $|I|=n-\left|\left\{X_{1}, \ldots, X_{k}\right\}\right|$, and since by the choice of $\lambda, 1-e^{-\lambda}=1 / 2$, then by Lemma 1.3 , with probability $\geq 3 / 4$

$$
n / 2-c^{\prime} \sqrt{n} \leq|I| \leq n / 2+c^{\prime} \sqrt{n},
$$

for some absolute constant $c^{\prime}>0$.

In the proof of (2.10) below we will refer to (2.4) rather than to (2.8), to be able to use directly parts of the proof of Theorem 2.1 .

Recall that $\left\{\varphi_{i}\right\}_{i \in I}=\left\{\left(\varphi_{j}\right)_{j \leq n} \backslash\left(X_{i}\right)_{i=1}^{k}\right\}$. As before, (2.4) implies that (2.6) is valid for all $y \in T \cap \rho S_{L_{2}^{n}}$. In turn, the upper estimate in the latter inequality implies that for all $y \in T \cap \rho S_{L_{2}^{n}}$,

$$
\sum_{j \in I}\left\langle\varphi_{j}, y\right\rangle^{2} \geq \sum_{j=1}^{n}\left\langle\varphi_{j}, y\right\rangle^{2}-\sum_{i=1}^{k}\left\langle X_{i}, y\right\rangle^{2} \geq \rho^{2}\left(1-\frac{4 k}{3 n}\right) .
$$

Exactly as before, (2.11) holds for all $y \in T$ for which $\|y\|_{L_{2}^{n}} \geq \rho$. Observe that since $\lambda=\log 2<3 / 4$ then $1-4 k /(3 n)>0$. Therefore, if $y \in T$ and $\left\langle\varphi_{j}, y\right\rangle=0$ for all $j \in I$, then $\|y\|_{L_{2}^{n}} \leq \rho$. Thus diam $\left((\operatorname{ker} \Gamma)^{\perp} \cap T\right) \leq \rho$ by the definition of $I$.

Let us also mention recent progress on random and non-random Euclidean sections of $L_{1}^{n}$ of proportional dimension generated by \pm 1 vectors. It has been initiated by G. Schechtman (see [Sch]) and followed by results in [LPRT], [AFMS] and [R2]. These results are based on a different model than the one we adopted in this section.

\section{Subsets of bounded orthonormal systems}

We now pass to the discussion of subspaces generated by subsets of bounded orthonormal systems in $L_{p}, p>2$. Our result is closely related to the $\Lambda_{p}$ problem solved by Bourgain ([B1]). Namely, we are loosing on the equivalence estimates (logarithmic instead of constant), although on the other hand, curiously enough, the sets we get have cardinality larger than expected, also by a logarithmic factor.

We shall use a general setting introduced at the beginning of Section 2 and in particular the semi-norm $\|\cdot\|_{X, k}$ on $L_{1}$ defined in (2.1).

Theorem 3.1. Let $\left(\varphi_{j}\right)_{j=1}^{n} \subset L_{2}$ be an orthonormal system with $\left\|\varphi_{j}\right\|_{L_{\infty}} \leq K$ for $1 \leq j \leq n$ and let $2<p<\infty$. There exists a subset $J \subset\{1, \ldots, n\}$ of cardinality $|J| \geq c_{p} K^{4 / p} n^{2 / p}(\log n)^{3}$ such that for all scalars $a_{1}, \ldots a_{n}$,

$$
\left\|\sum_{j \in J} a_{j} \varphi_{j}\right\|_{L_{p}} \leq K C_{p}(\log n)^{3 / 2}\left(\sum_{j \in J} a_{j}^{2}\right)^{1 / 2}
$$

where $c_{p}$ and $C_{p}$ depend only on $p$. 
The proof requires a lemma analogous to Lemma 2.2.

Lemma 3.2. For every $1<q \leq 2$ there exists a constant $C_{q}$ depending only on $q$ such that for every $k$ and $X_{1}, \ldots, X_{k}$ with $\left\|X_{i}\right\|_{L_{\infty}} \leq K$,

$$
\gamma_{2}\left(B_{L_{q}},\|\cdot\|_{X, k}\right) \leq K C_{q}(\log (1+k))^{3 / 2} .
$$

Proof. The same argument as in Lemma 2.2 shows that

$$
\gamma_{2}\left(B_{L_{q}},\|\cdot\|_{X, k}\right) \leq C \sum_{\ell=1}^{k} \frac{e_{\ell}\left(S^{*}\right)}{\sqrt{\ell}},
$$

where $C$ is an absolute constant, the operator $S: \ell_{1}^{k} \rightarrow L_{p}$ is defined by $S\left(e_{i}\right)=X_{i}$ for every $i=1, \ldots, k$, and $p=q /(q-1)$.

Since for $1<p<\infty L_{p}$ is uniformly convex then a duality result from [BPST] (Theorem 1) yields that there is a constant $c_{p}$ depending only on $p$ such that

$$
\sum_{\ell=1}^{k} \frac{e_{\ell}\left(S^{*}\right)}{\sqrt{\ell}} \leq c_{p} \sum_{\ell=1}^{k} \frac{e_{\ell}(S)}{\sqrt{\ell}} .
$$

For $p \geq 2, L_{p}$ has type 2 (with the type 2 constant depending on $p$ ), so using Proposition 1 of Carl [C], it follows that for $\ell \leq k$,

$$
e_{\ell}(S) \leq C_{p}^{\prime}\|S\| \sqrt{\frac{\log (1+k / \ell)}{\ell}} .
$$

This inequality together with $\|S\| \leq \max _{1 \leq i \leq k}\left\|X_{i}\right\|_{L_{p}} \leq K$ give an estimate of the right-hand side of (3.1). We conclude that

$$
\gamma_{2}\left(B_{L_{q}},\|\cdot\|_{X, k}\right) \leq K C_{p}(\log (1+k))^{3 / 2},
$$

where $C_{p}$ depends on $p$ (and hence on $q$ ) only.

Proof of Theorem 3.1 Define the random vector $X$ by $X=\varphi_{j}$ with probability $1 / n$ for $1 \leq j \leq n$. Fix $k \leq n$ to be determined later and let $X_{1}, \ldots, X_{k}$ be independent copies of $X$.

For $k \geq 1$ set $B_{2}^{k}=\left\{a \in \mathbb{C}^{k}: \sum_{i=1}^{k}\left|a_{i}\right|^{2} \leq 1\right\}$. We will prove that there exists $k \geq K^{4 / p} c_{p} n^{2 / p}(\log n)^{3}$ such that

$$
\mathbb{E} \sup _{a \in B_{2}^{k}}\left\|\sum_{i=1}^{k} a_{i} X_{i}\right\|_{L_{p}} \leq K C_{p}(\log n)^{3 / 2} .
$$

The theorem will follow by applying Lemma 1.3 for $k_{0}=\left[K^{4 / p} c_{p} n^{2 / p}(\log n)^{3}\right]+1$ and observing that (in the notation of that lemma) with high probability, $\alpha_{k_{0}} \geq$ $k_{0} / 2$. Thus, the set $\left\{X_{1}, \ldots, X_{k_{0}}\right\}$ contains at least $k_{0} / 2$ distinct vectors $\varphi_{j}$ 's.

Passing to the proof of $(3.2)$,

$$
\mathbb{E} \sup _{a \in B_{2}^{k}}\left\|\sum_{i=1}^{k} a_{i} X_{i}\right\|_{L_{p}}=\mathbb{E} \sup _{y \in B_{L_{q}}}\left(\sum_{i=1}^{k}\left\langle X_{i}, y\right\rangle^{2}\right)^{1 / 2} .
$$


Next, apply Theorem 1.2 for $F=B_{L_{q}}$. Recall that $d_{\infty, k}(f, g)=\|f-g\|_{X, k}$ and note that the latter expectation is less than or equal to

$$
\begin{aligned}
\mathbb{E} \sup _{y \in B_{L_{q}}} & \left(\sum_{i=1}^{k}\left\langle X_{i}, y\right\rangle^{2}-k \mathbb{E}\langle X, y\rangle^{2}\right)^{1 / 2}+\sqrt{k} \sigma_{F} \\
\leq & \leq C\left(\left(\max \left(\sqrt{k} \sigma_{F} U_{k}, U_{k}^{2}\right)\right)^{1 / 2}+\sqrt{k} \sigma_{F}\right) .
\end{aligned}
$$

By Lemma 3.2, $U_{k} \leq K C_{p}(\log k)^{3 / 2}$, and since the functions $\varphi_{i}$ are bounded in $L_{\infty}$ it is evident that for every $\left(a_{i}\right)_{i=1}^{n} \in \mathbb{C}^{n}$,

$$
\left\|\sum_{i=1}^{n} a_{i} \varphi_{i}\right\|_{L_{\infty}} \leq K \sum_{i=1}^{n}\left|a_{i}\right| \leq K \sqrt{n}\left(\sum_{i=1}^{n}\left|a_{i}\right|^{2}\right)^{1 / 2} .
$$

Moreover, since $\left(\varphi_{i}\right)_{i=1}^{n}$ are orthonormal in $L_{2}$ then

$$
\left\|\sum_{i=1}^{n} a_{i} \varphi_{i}\right\|_{L_{2}}=\left(\sum_{i=1}^{n}\left|a_{i}\right|^{2}\right)^{1 / 2}
$$

and by Hölder inequality it follows that for every $p \geq 2$ and every $\left(a_{i}\right)_{i=1}^{n} \in \mathbb{C}^{n}$,

$$
\left\|\sum_{i=1}^{n} a_{i} \varphi_{i}\right\|_{L_{p}} \leq K^{1-2 / p} n^{1 / 2-1 / p}\left(\sum_{i=1}^{n}\left|a_{i}\right|^{2}\right)^{1 / 2} .
$$

Considering the operator $S: \ell_{2}^{n} \rightarrow L_{p}$ defined by $S\left(e_{i}\right)=\varphi_{i}$ for $i=1, \ldots, n$ the above inequality means that $\|S\| \leq K^{1-2 / p} n^{1 / 2-1 / p}$. By duality,

$$
\begin{aligned}
\sigma_{F}:=\sup _{y \in B_{L_{q}}}\left(\mathbb{E}\langle X, y\rangle^{2}\right)^{1 / 2} & =n^{-1 / 2} \sup _{y \in B_{L_{q}}}\left(\sum_{i=1}^{n}\left\langle\varphi_{i}, y\right\rangle^{2}\right)^{1 / 2} \\
& =n^{-1 / 2}\left\|S^{*}\right\| \leq K^{1-2 / p} n^{-1 / p} .
\end{aligned}
$$

Therefore, by Theorem 1.2,

$$
\begin{gathered}
\mathbb{E} \sup _{a \in B_{2}^{k}}\left\|\sum_{i=1}^{k} a_{i} X_{i}\right\|_{L_{p}} \\
\leq C\left(\left(\max \left(\sqrt{k} K^{2-2 / p} c_{p} n^{-1 / p}(\log k)^{3 / 2}, K^{2} c_{p}^{2}(\log k)^{3}\right)\right)^{1 / 2}\right. \\
\left.+\sqrt{k} K^{1-2 / p} n^{-1 / p}\right) .
\end{gathered}
$$

Setting $k$ to be the smallest integer greater than $K^{4 / p} C_{p} n^{2 / p}(\log n)^{3}$, we obtain the claimed inequality (3.2).

We conclude this section with several remarks: 
1. The same argument as in the proof of Theorem 3.1 shows that an analogous result holds for all dimensions $k \geq k_{0}:=\left[c_{p} K^{4 / p} n^{2 / p}(\log n)^{3}\right]+1$. More precisely, under the hypothesis of Theorem 3.1, for every $k_{0} \leq k \leq n$ there is a subset $J \subset\{1, \ldots, n\}$ of cardinality $|J| \geq k / 2$ such that for all scalars $a_{1}, \ldots a_{n}$

$$
\left\|\sum_{j \in J} a_{j} \varphi_{j}\right\|_{L_{p}} \leq K^{1-2 / p} C_{p} \sqrt{k} n^{-1 / p}\left(\sum_{j \in J} a_{j}^{2}\right)^{1 / 2},
$$

where $C_{p}$ depends on $p$ only. A careful reading of the consequences of the main result of the paper of Bourgain [B2] (using equation (3.4) and (3.5) in [B2]) shows that this result is in fact valid for every $k \geq c(p, K) n^{2 / p}$ where $c(p, K)$ depends on $p$ and $K$.

2. The estimate of Theorem 3.1, as well as (3.3), are, in general, asymptotically best possible. Indeed, this is the case, for example, when $\left(\varphi_{j}\right)_{j=1}^{n}$ is an orthonormal system in $L_{2}^{n}$ with $\left\|\varphi_{j}\right\|_{L_{\infty}^{n}} \leq K$ for $1 \leq j \leq n$ (as in the setting of Theorem 2.1). Clearly, (3.3) implies that $E:=\operatorname{span}\left\{\varphi_{j}\right\}_{j \in J}$ is a $d$-Euclidean subspace of $L_{p}^{n}$ with $\operatorname{dim} E=|J|$ (where $d:=K^{1-2 / p} C_{p} \sqrt{k} n^{-1 / p}$ ). Thus by a well-known argument on Euclidean subspaces of $L_{p}^{n}$ (see e.g., [MS], 5.6), $\sqrt{|J|} \leq C_{p}^{\prime} d n^{1 / p}$, for some $C_{p}^{\prime}$ depending on $p$ only.

3. B. Kashin pointed out to us that, in the particular case of the trigonometric system, Theorem 3.1 is related to the notion of the trigonometric $n$-width introduced by Ismagilov in [I] as a "restricted Kolmogorov width," and extensively studied in approximation theory in the 1980's for various spaces of smooth functions. For a compact subset $B$ of $L_{q}[0,2 \pi]$ and $\ell \geq 1$, the $\ell$ 'th trigonometric width is defined by

$$
d_{\ell}^{T}\left(B, L_{q}\right):=\inf _{E \in \mathcal{T}_{\ell}} \sup _{x \in B} \inf _{e \in E}\|x-e\|_{L_{q}},
$$

where $\mathcal{T}_{\ell}$ ranges over the set of all subspaces $E$ spanned by $\ell$ elements of the trigonometric system. By duality, Theorem 3.1 and Remark 1 above in the case of the trigonometric system, provide an estimate for $d_{\ell}^{T}\left(B, L_{2}\right)$ where $B$ is the unit $L_{p^{\prime}}$-ball in the space of trigonometric polynomials of degree $n$ and $\ell=n-k$ for $k \geq c_{p} n^{2 / p}\left(\right.$ and $\left.p^{\prime}=p /(p-1)\right)$.

\section{Acknowledgement}

The authors would like to thank Boris Kashin for his comments on trigonometric $n$-widths and suggestions elaborated in Remarks 1 and 3 at the end of Section 3. Part of the work on this article was conducted during the Trimesters "Phenomena in High Dimensions" held at the Schrödinger Institute, Vienna in the summer of 2005 and at the Centre Emile Borel, Institut Henri Poincaré, Paris in the Spring of 2006. We are grateful to these Institutes for their hospitality and the excellent working atmosphere it provided. 


\section{References}

[AFMS] S. Artstein-Avidan, O. Friedland, V. Milman \& S. Sodin, Polynomial bounds for large Bernoulli sections of the cross polytope, preprint.

[B1] J. Bourgain, Bounded orthogonal systems and the $\Lambda(p)$-set problem, Acta Math. 162 (1989), no. 3-4, 227-245.

[B2] J. Bourgain, On $\Lambda(p)$-subsets of squares, Israel J. Math. 67 (1989), no. 3, 291311.

[BPST] J. Bourgain, A. Pajor, S. Szarek \& N. Tomczak-Jaegermann, On the duality problem for entropy numbers of operators, in Geometric aspects of functional analysis (1987-88), 50-63, Lecture Notes in Math., 1376, Springer, Berlin, 1989.

[C] B. Carl, Inequalities of Bernstein-Jackson-type and the degree of compactness of operators in Banach spaces, Ann. Inst. Fourier (Grenoble) 35 (1985), no. 3, 79-118.

[GZ] E. Giné \& J. Zinn, Some limit theorems for empirical processes, Ann. Probab. 12 (1984), no. 4, 929-998.

[GR] O. Guédon \& M. Rudelson, $L_{p}$ moments of random vectors via majorizing measures, Adv. Math. 208 (2007), no. 2, 798-823.

[I] R. S. Ismagilov, Diameters of sets in normed linear spaces, and the approximation of functions by trigonometric polynomials, Uspehi Mat. Nauk 29 (1974), no. 3(177), 161-178. (Russian)

[LPRT] A. Litvak, A. Pajor, M. Rudelson \& N. Tomczak-Jaegermann, Smallest singular value of random matrices and geometry of random polytopes, Adv. Math. 195 (2005), no. 2, 491-523.

$[\mathrm{K}] \quad$ B. Kashin, The widths of certain finite-dimensional sets and classes of smooth functions, Izv. Akad. Nauk SSSR Ser. Mat. 41 (1977), no. 2, 334-351, 478.

[MS] V. Milman \& G. Schechtman, Asymptotic theory of finite dimensional normed spaces, Lect. Notes in Math. 1200, Springer 1986.

$[\mathrm{P}] \quad$ G. Pisier, The volume of convex bodies and Banach space geometry, Cambridge Univ. Press, Cambridge, 1989.

[R1] M. Rudelson, Private communication

[R2] M. Rudelson, Lower estimates for the singular values of random matrices, C. R. Math. Acad. Sci. Paris 342 (2006), no. 4, 247-252.

[RV] M. Rudelson \& R. Vershynin, Sparse reconstruction by convex relaxation: Fourier and Gaussian measurements, CISS 2006 (40th Annual Conference on Information Sciences and Systems).

[Sch] G. Schechtman, Special orthogonal splittings of $L_{1}^{2 k}$, Israel J. Math. 139 (2004), 337-347.

[S] S. Szarek, On Kashin's almost Euclidean orthogonal decomposition of $l_{n}^{1}$, Bull. Acad. Polon. Sci. Sér. Sci. Math. Astronom. Phys. 26 (1978), no. 8, 691-694.

[ST] S. Szarek \& N. Tomczak-Jaegermann, On nearly Euclidean decomposition for some classes of Banach spaces. Compositio Math. 40 (1980), no. 3, 367-385.

[Sz] E. Szemerédi, On sets of integers containing no $k$ elements in arithmetic progression, Acta. Arith., 27 (1975), 299-345. 
[Ta1] M. Talagrand, The generic chaining, Springer, Berlin, 2005.

[Ta2] M. Talagrand, Selecting a proportion of characters, Israel J. Math. 108 (1998), 173-191.

Olivier Guédon

Institut de Mathématiques de Jussieu

Université Pierre et Marie Curie

Paris 6

4 place Jussieu

75005 Paris

France

e-mail: guedon@math.jussieu.fr

Shahar Mendelson

Centre for Mathematics and its Applications

The Australian National University

Canberra ACT 0200

Australia

e-mail: shahar.mendelson@anu.edu.au

and

Department of Mathematics

Technion I.I.T.

Haifa 3200

Israel

Alain Pajor

Équipe d'Analyse et Mathématiques Appliquées

Université de Marne-la-Vallée

5 boulevard Descartes

Champs sur Marne

77454 Marne-la-Vallée Cedex 2

France

e-mail: Alain.Pajor@univ-mlv.fr

Nicole Tomczak-Jaegermann

Dept. of Math. and Stat. Sciences

University of Alberta

Edmonton

T6G 2G1 Alberta

Canada

e-mail: nicole.tomczak@ualberta.ca

Received 4 September 2006; accepted 18 October 2006 